\title{
Microstructural Activation of a Topochemical Reduction Reaction
}

\author{
Zhilin Liang, Midori Amano Patino, Mylène Hendrickx, Joke Hadermann, and Michael A. Hayward* \\ Cite This: ACS Org. Inorg. Au 2022, 2, 75-82 \\ Read Online
}

ACCESS |

Wll Metrics \& More

Article Recommendations

SI Supporting Information

ABSTRACT: The progress of the topochemical reduction reaction that converts $\mathrm{LaSrNiRuO}_{6}$ into $\mathrm{LaSrNiRuO}_{4}$ depends on the synthesis conditions used to prepare the oxidized phase. Samples of $\mathrm{LaSrNiRuO}_{6}$ that have been quenched from high temperature can be readily and rapidly converted into LaSrNiR$\mathrm{uO}_{4}$. In contrast, samples that have been slow-cooled cannot be completely reduced. This reactivity difference is attributed to the differing microstructures of the quenched and slow-cooled samples, with the former having much smaller average crystalline domain sizes and larger lattice strains than the latter. A mechanism to explain this effect is presented, in which the greater "plasticity"

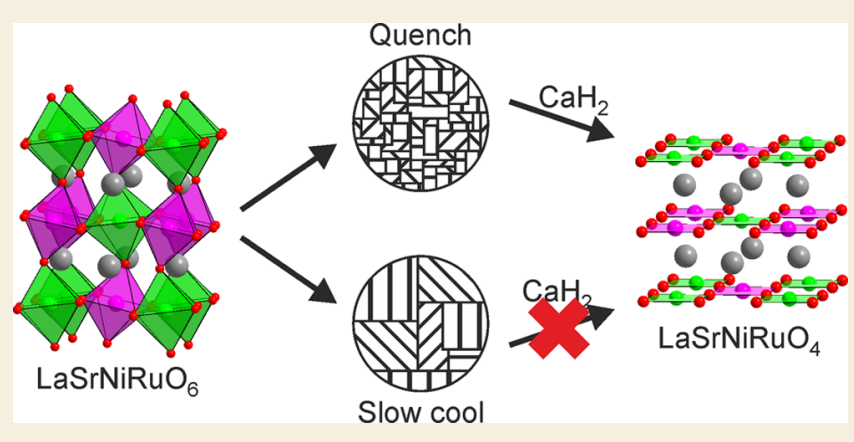
of small crystalline domains helps lower the activation energy of the reduction reaction. In addition, we propose that the enhanced lattice strain in quenched samples also acts to destabilize the host phase, further enhancing reactivity. These observations suggest that the microstructure of a material can be used to "activate" topochemical reactions in the solid state, expanding the scope of phases that can be prepared by this type of reaction.

KEYWORDS: Topochemical reduction, Transition-metal oxides, Solid-state microstructure, Solid-state synthesis, Phase transitions

\section{INTRODUCTION}

Solid-state syntheses performed under "conventional" hightemperature conditions tend to form products that are "selected" on the basis of their thermodynamic stability. This behavior can be attributed to the large energetic barriers that oppose diffusion in the solid state. These large barriers not only necessitate the use of high reaction temperatures but also tend to dominate the activation energies of the many possible reactions that could occur between solid components. As a result, these competing reactions establish an equilibrium at the high temperatures required for reactions to proceed, yielding products that are selected on the basis of their thermodynamic stability. As a consequence, there are many metastable phases that cannot be prepared under these hightemperature conditions.

Low-temperature topochemical (structure conserving) reactions can overcome the dominance of thermodynamic product selection in the solid state, by utilizing the observation that while the energetic barriers to diffusion are high in solids, they do not apply uniformly. There are many solid compounds in that a subset of the species present are more mobile than the "host framework" in that they are embedded. This difference in mobility amounts to a difference in reactivity, allowing the more mobile species to be removed, inserted, or exchanged within the immobile framework, facilitating preparation of metastable phases with chemical compositions and structures that can be considered as modifications of the original host phase. ${ }^{1}$
This topochemical approach is particularly valuable in the preparation of complex transition-metal oxides, as conventional high-temperature synthesis techniques tend to prepare phases with transition-metal cations in the limited subset of oxidationstate/coordination-geometry combinations that are the most thermodynamically stable. Topochemical manipulations, particularly when applied to modify the anion lattices of phases, allow the range of oxidation-state/coordination-environment combinations that can be stabilized within extended oxide phases to be increased dramatically. ${ }^{2-12}$

While the products of high-temperature reactions can only be changed by modifying the relative thermodynamic stability of competing product phases, the kinetic control that is in operation during topochemical reactions offers many opportunities to direct and modify the product phases formed, by tuning the many features of the reagents and reaction conditions that come together to determine reaction rates. Recently, we reported the preparation of $\mathrm{LaSrNiRuO}_{4}$ via the topochemical reduction of $\mathrm{LaSrNiRuO}_{6}$, as shown in Figure 1. ${ }^{13}$ While investigating the preparation of this phase, we observed a highly unusual behavior, in which the reactivity of the "host" $\mathrm{LaSrNiRuO}_{6}$ phase depends on the exact conditions

Received: October 6, 2021

Revised: October 29, 2021

Accepted: November 1, 2021

Published: November 15, 2021

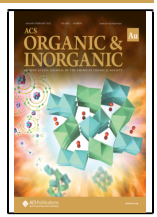




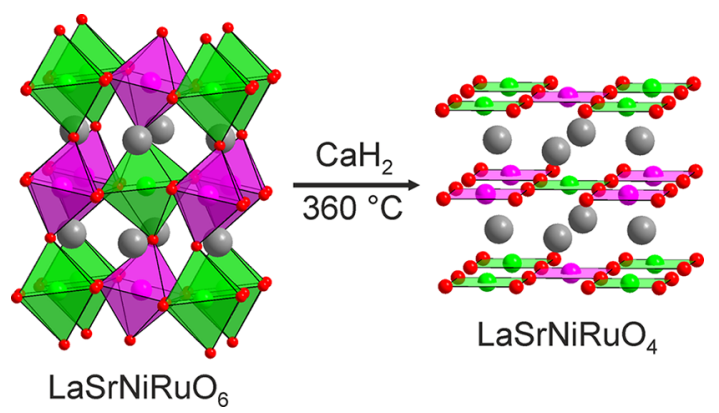

Figure 1. Reduction of $\mathrm{LaSrNiRuO}_{6}$ with $\mathrm{CaH}_{2}$ yields $\mathrm{LaSrNiRuO}_{4}$ via a topochemical reduction reaction. $\mathrm{La} / \mathrm{Sr}, \mathrm{Ni}, \mathrm{Ru}$, and $\mathrm{O}$ positions are represented by gray, green, purple, and red spheres, respectively.

under which the material was prepared. Samples of $\mathrm{LaSrNiRuO}_{6}$ rapidly cooled from high temperature could be readily converted to $\mathrm{LaSrNiRuO}_{4}$ by reaction with $\mathrm{CaH}_{2}$. In contrast, samples of $\mathrm{LaSrNiRuO}_{6}$ that had been cooled slowly could not be completely reduced to form $\mathrm{LaSrNiRuO}_{4}$. The differing reactivity of slow-cooled and quenched samples suggests there is an additional method that can be used to control the progress of topochemical reactions, which we investigate further here.

\section{EXPERIMENTAL SECTION}

\section{Synthesis of LaSrNiRuO 6}

Samples of $\mathrm{LaSrNiRuO}_{6}$ were prepared via a citrate gel method. Suitable stoichiometric ratios of $\mathrm{La}_{2} \mathrm{O}_{3}$ (99.999\%), $\mathrm{SrCO}_{3}$ (99.99\%), $\mathrm{Ni}$ powder (99.996\%), and $\mathrm{RuO}_{2}\left(99.99 \%\right.$ dried at $\left.800{ }^{\circ} \mathrm{C}\right)$ were dissolved in a minimum quantity of $6 \mathrm{M}$ nitric acid. Then, $3 \mathrm{~mol}$ equivalents of citric acid and $5 \mathrm{~mL}$ of analar ethylene glycol were added, and the solution was heated with constant stirring. The gels thus formed were subsequently ground into a fine powder, placed in an alumina crucible, and heated at $1{ }^{\circ} \mathrm{C} \min ^{-1}$ to $900{ }^{\circ} \mathrm{C}$ in air. The powders were then reground, pressed into $13 \mathrm{~mm}$ pellets, and then heated at $1100{ }^{\circ} \mathrm{C}$ in air for two periods of $24 \mathrm{~h}$. At the end of the final heating period, samples were either cooled to room temperature at a rate of $4{ }^{\circ} \mathrm{C} \mathrm{min}^{-1}$ (subsequently referred to as slow-cooled samples) or removed from the furnace and rapidly transferred to a dry-ice-cooled alumina crucible and allowed to rapidly cool (subsequently referred to as quenched samples).

\section{Reduction of $\mathrm{LaSrNiRuO}_{6}$ to $\mathrm{LaSrNiRuO}_{4}$}

Samples of $\mathrm{LaSrNiRuO}_{6}$ were reduced by reacting with $4 \mathrm{~mol}$ equivalents of $\mathrm{CaH}_{2}$ at $360{ }^{\circ} \mathrm{C}$ in a "venting" apparatus described previously. ${ }^{14}$ After the reaction, calcium-containing phases were removed from samples by washing with a $0.05 \mathrm{M}$ solution of $\mathrm{NH}_{4} \mathrm{Cl}$ in methanol and then a further $4 \times 80 \mathrm{~mL}$ aliquots of clean methanol before being dried under vacuum.

\section{Characterization}

Laboratory X-ray powder diffraction data were collected from samples contained in gastight sample holders using a PANalytical X'Pert diffractometer incorporating an X'celerator position sensitive detector (monochromatic $\mathrm{Cu} \mathrm{K}_{\alpha 1}$ radiation). High-resolution synchrotron Xray powder diffraction (SXRD) data were collected using the I11 instrument at the Diamond Light Source Ltd. Diffraction patterns were collected using Si-calibrated X-rays with an approximate wavelength of $0.825 \AA$ from samples sealed in $0.3 \mathrm{~mm}$ diameter borosilicate glass capillaries. Rietveld refinements were performed using TOPAS Academic (V6). ${ }^{15}$ Thermogravimetric measurements were performed by heating powder samples at a rate of $5{ }^{\circ} \mathrm{C} \min ^{-1}$ under a $10 \% \mathrm{H}_{2}$ in $\mathrm{N}_{2}$ atmosphere, using a Mettler Toledo MX1 thermogravimetric microbalance. Scanning electron microscopy (SEM) images were collected using a Thermo Fisher Quanta FEG
250 microscope at $10 \mathrm{kV}$. The particle-size analysis was performed in DigitalMicrograph.

\section{RESULTS AND DISCUSSION}

\section{Reactivity of LaSrNiRuO 6 with $\mathrm{CaH}_{2}$}

Two separate samples of $\mathrm{LaSrNiRuO}_{6}$, one prepared by a slowcooled method and one by a quenched method, were ground together with $4 \mathrm{~mol}$ equivalents of $\mathrm{CaH}_{2}$ and then heated at $360{ }^{\circ} \mathrm{C}$ for three periods of $48 \mathrm{~h}$, with intermediate regrinding. $\mathrm{X}$-ray powder diffraction data (Figure 2) indicated that the

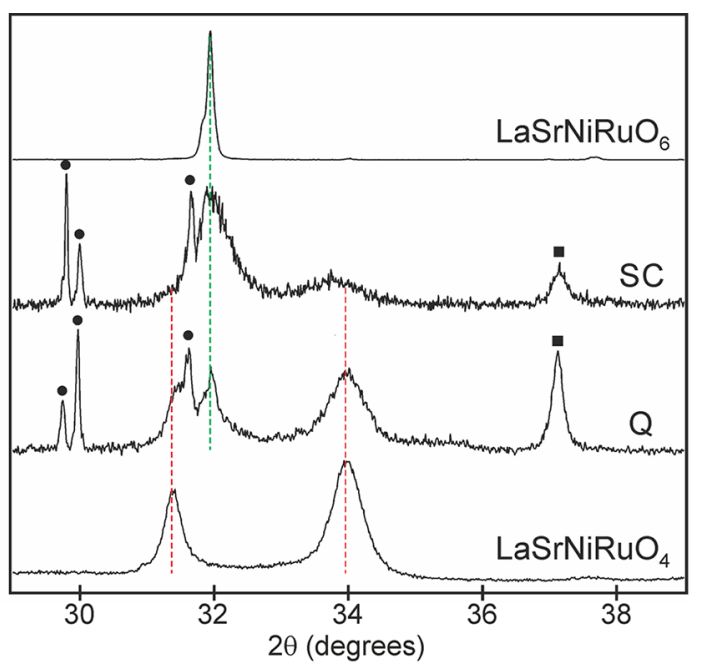

Figure 2. X-ray powder diffraction data collected from the products of reaction between slow-cooled (SC) and quenched (Q) $\mathrm{LaSrNiRuO}_{6}$ and $\mathrm{CaH}_{2}$ after three periods of heating at $360{ }^{\circ} \mathrm{C}$. Analogous data collected from $\mathrm{LaSrNiRuO}_{6}$ and $\mathrm{LaSrNiRuO}_{4}$ are plotted for comparison. Solid circles indicate peak positions of $\mathrm{CaH}_{2}$, and solid squares indicate peak positions of $\mathrm{CaO}$.

products of both reactions were mixtures of pseudocubic $\mathrm{LaSrNiRuO}_{6-x}$, pseudotetragonal $\mathrm{LaSrNiRuO}_{4+x}, \mathrm{CaH}_{2}$, and $\mathrm{CaO}$. The ratio of $\mathrm{LaSrNiRuO}_{6-x} / \mathrm{LaSrNiRuO}_{4+x}$ in the samples can be crudely quantified by comparing the area of the overlapping $200 / 020 / 112$ reflections $\left(2 \theta \approx 32^{\circ}\right)$ of $\mathrm{LaSrNiRuO}_{6-x}$ with that of the 112 reflection $\left(2 \theta \approx 34^{\circ}\right)$ of $\mathrm{LaSrNiRuO}_{4+x}$ and normalizing for their relative multiplicities. This analysis reveals a $\mathrm{LaSrNiRuO}_{6-x} / \mathrm{LaSrNiRuO}_{4+x}$ ratio of 74(1):26(1) for the slow-cooled sample and 29(1):71(1) for the quenched sample, demonstrating that the quenched sample reacted faster and further than the slow-cooled sample.

Two further heating periods of $48 \mathrm{~h}$ at $360{ }^{\circ} \mathrm{C}$ converted the quenched sample completely into $\mathrm{LaSrNiRuO}_{4}$. In contrast, further heating of the slow-cooled sample at $360{ }^{\circ} \mathrm{C}$ led to no further reaction, with the ratio of $\mathrm{LaSrNiRuO}_{6-x} / \mathrm{LaSrNiR}$ $\mathrm{uO}_{4+x}$ remaining unchanged. Raising the temperature of reaction to $370{ }^{\circ} \mathrm{C}$ led to sample decomposition via nontopochemical reactions, and the formation of poorly crystalline $\mathrm{Ni}, \mathrm{Ru}, \mathrm{La}_{2} \mathrm{O}_{3}$, and $\mathrm{SrO}$. These results demonstrate that there is a significant difference in the reactivity of the slowcooled and quenched samples with $\mathrm{CaH}_{2}$. Furthermore, in contrast to quenched samples, there appear to be no conditions under which a slow-cooled sample of $\mathrm{LaSrNiRuO}_{6}$ can be converted into a phase-pure sample of $\mathrm{LaSrNiRuO}_{4}$ by reduction with $\mathrm{CaH}_{2}$. 
Structural Characterization of Slow-Cooled and Quenched LaSrNiRuO

X-ray powder diffraction patterns collected from both slowcooled and quenched samples of $\mathrm{LaSrNiRuO}_{6}$ prior to reduction could be readily indexed using the monoclinic unit cell reported for the phase. ${ }^{16}$ Refinement of a model based on the reported structure of $\mathrm{LaSrNiRuO}_{6}$ (space group $\left.P 2_{1} / n\right)^{16}$ against these diffraction data proceeded smoothly. The detailed results of the structural refinement (Table S1 in the Supporting Information) reveal the structures of the slowcooled and quenched samples of $\mathrm{LaSrNiRuO}_{6}$ are indistinguishable. Furthermore, thermogravimetric analysis (Figure S1, Supporting Information) indicated both quenched and slowcooled samples were oxygen-stoichiometric. Combined, these observations indicate that the slow-cooled and quenched samples of $\mathrm{LaSrNiRuO}_{6}$ are extremely similar with respect to their bulk crystal structures and chemical compositions, so these features are unlikely to be the origin of their differing reactivity.

\section{Microstructural Characterization of Slow-Cooled and Quenched LaSrNiRuO}

Although the refined structures of the slow-cooled and quenched samples are indistinguishable, direct comparison of the X-ray powder diffraction data collected from the two samples reveals all the diffraction peaks in the data set from the quenched sample are significantly broader than the corresponding features from the slow-cooled sample, as can be seen in Figure $3 a, b$ and a plot of the diffraction peak full-width at half-maximum (fwhm) against $2 \theta$ shown in Figure $3 \mathrm{c}$. Separation of the fwhm $2 \theta$-dependence into contributions from crystal domain size and lattice strain (described in detail in the Supporting Information) reveals that the quenched sample has a smaller average particle/domain size (quenched:

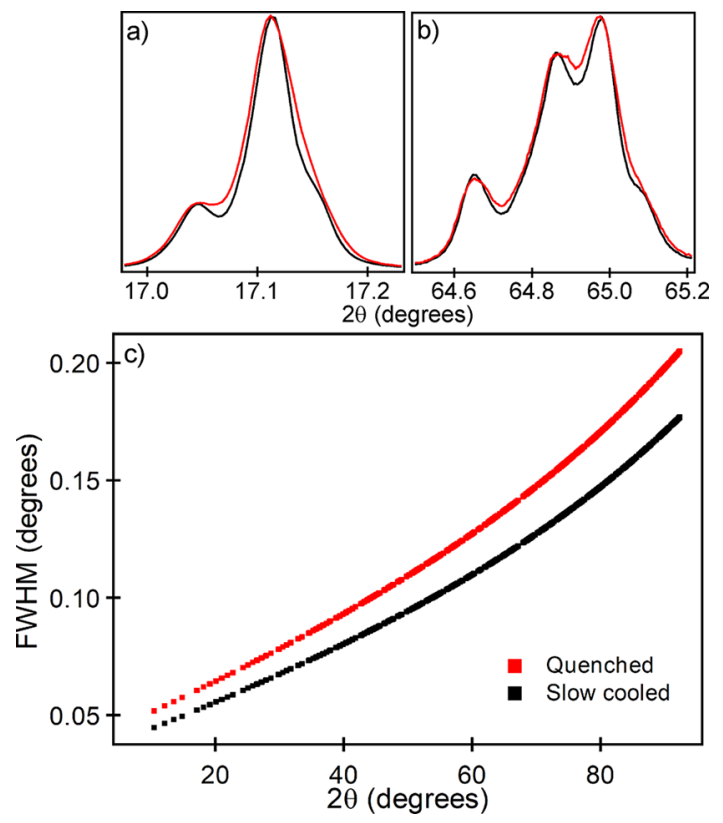

Figure 3. Plot of selected regions of the SXRD data from quenched (red) and slow-cooled (black) samples of $\mathrm{LaSrNiRuO}_{6}$ at (a) $2 \theta \approx$ $17.1^{\circ}$ and (b) $2 \theta \approx 64.9^{\circ}$. (c) A plot of fwhm against $2 \theta$ of SXRD diffraction peaks collected from slow-cooled and quenched samples of $\mathrm{LaSrNiRuO}_{6}$.
122.7(2) nm; slow-cooled: $142.6(3) \mathrm{nm}$ ) and a lattice strain which is $30 \%$ higher than the slow-cooled sample.

To further examine the microstructure of the samples, we measured the average particle size of slow-cooled and quenched samples of $\mathrm{LaSrNiRuO}_{6}$ via the analysis of SEM images, as described in detail in the Supporting Information. This analysis yielded values of 184(3) $\mathrm{nm}$ and 214(5) $\mathrm{nm}$ for the particle sizes of quenched and slow-cooled samples, respectively. These values are about $50 \%$ larger than those extracted from the SXRD data, suggesting that the particles of $\mathrm{LaSrNiRuO}_{6}$ contain multiple crystalline domains. In addition, close inspection of the particle-size distributions revealed that a significant fraction of the particles in the slow-cooled sample have diameters greater than $400 \mathrm{~nm}(\sim 13 \%)$, while there are almost no particles of this size in the quenched sample $(<4 \%)$.

This microstructural analysis of $\mathrm{LaSrNiRuO}_{6}$ clearly shows that the quenched sample contains smaller, more strained crystal domains than the slow-cooled sample. We propose it is this microstructural difference, rather than some compositional difference, which is responsible for the differing reactivity of slow-cooled and quenched samples of $\mathrm{LaSrNiRuO}_{6}$, as described below.

The role of the postsynthesis cooling rate in defining the crystalline domain size and lattice strain of $\mathrm{LaSrNiRuO}_{6}$ samples appears to be related to the structural phase transition this material exhibits at around $400{ }^{\circ} \mathrm{C},{ }^{16}$ in which the arrangement of cooperatively tilted $\mathrm{NiO}_{6}$ and $\mathrm{RuO}_{6}$ octahedra changes from a high-temperature scheme described as $a^{-} a^{-} a^{-}$ in Glazer notation (space group $R-3),{ }^{17,18}$ to an $a^{-} a^{-} c^{+}$twisting pattern at low temperature (space group $P 2_{1} / n$ ). To further investigate this behavior, we performed a series of quenching experiments, in which pellets of $\mathrm{LaSrNiRuO}_{6}$ were heated to $1100{ }^{\circ} \mathrm{C}$ (the synthesis temperature) and then slow-cooled to the "quenching temperature" indicated in Figure 4 and held

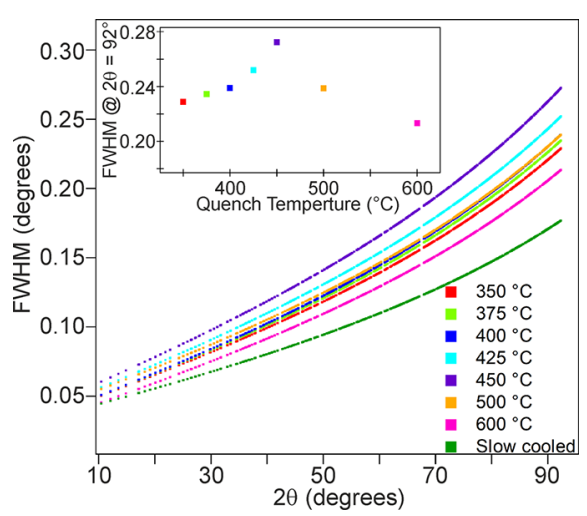

Figure 4. Plot of fwhm against $2 \theta$ of SXRD data collected from samples of $\mathrm{LaSrNiRuO}_{6}$ quench from different temperatures. Inset shows the fwhm of the diffraction peak at $2 \theta=92^{\circ}$ as a function of quenching temperature.

there for $2 \mathrm{~h}$. The samples were then thermally quenched by rapidly removing them from the furnace and transferring them to a dry-ice-cooled alumina crucible. SXRD data collected from these samples, after they had been ground into powder, were fit by the $P 2_{1} / n$ symmetry model of $\mathrm{LaSrNiRuO}_{6}$ to yield lattice parameters indistinguishable from the other samples of $\mathrm{LaSrNiRuO}_{6}$ prepared in this study. Figure 4 shows a plot of the SXRD diffraction peak fwhm as a function of $2 \theta$ for the different quenched samples, with the inset showing the fwhm 
of the diffraction peak at $2 \theta=92^{\circ}$ for samples as a function of quenching temperature. These data show that rapidly quenching from $450{ }^{\circ} \mathrm{C}$ yields samples with the broadest diffraction peaks and that all samples quenched in the range $350<T /{ }^{\circ} \mathrm{C}<600$ have significantly broadened diffraction peaks compared to a slow-cooled sample.

The influence of quenching on the microstructure of samples can be rationalized by noting there is no group/ subgroup relation between the $R-3$ space group of the hightemperature phase and the $P 2_{1} / n$ space group of the lowtemperature phase, and as a consequence, there is no preference for any of the six possible orientations of the monoclinic cell axes of the low-temperature phase with respect to the rhombohedral cell axes of the high-temperature phase. Described in terms of crystal structure, one of the three $a^{-}$outof-phase tilts becomes a $c^{+}$in-phase tilt at the phase transition. This in-phase tilt could be aligned along either the $x$-, $y$-, or $z$ axes of the aristotype perovskite framework and be either "clockwise" or "anticlockwise" in sense, giving six equivalent possibilities. Thus, when the structural symmetry of the $\mathrm{LaSrNiRuO}_{6}$ framework is lowered throughout the sample on cooling, a patchwork of small domains of the monoclinic phase is formed, each with a different cell axis orientation (orientation/sense of the $c^{+}$tilt) compared to its neighbors, as shown in Figure 5.

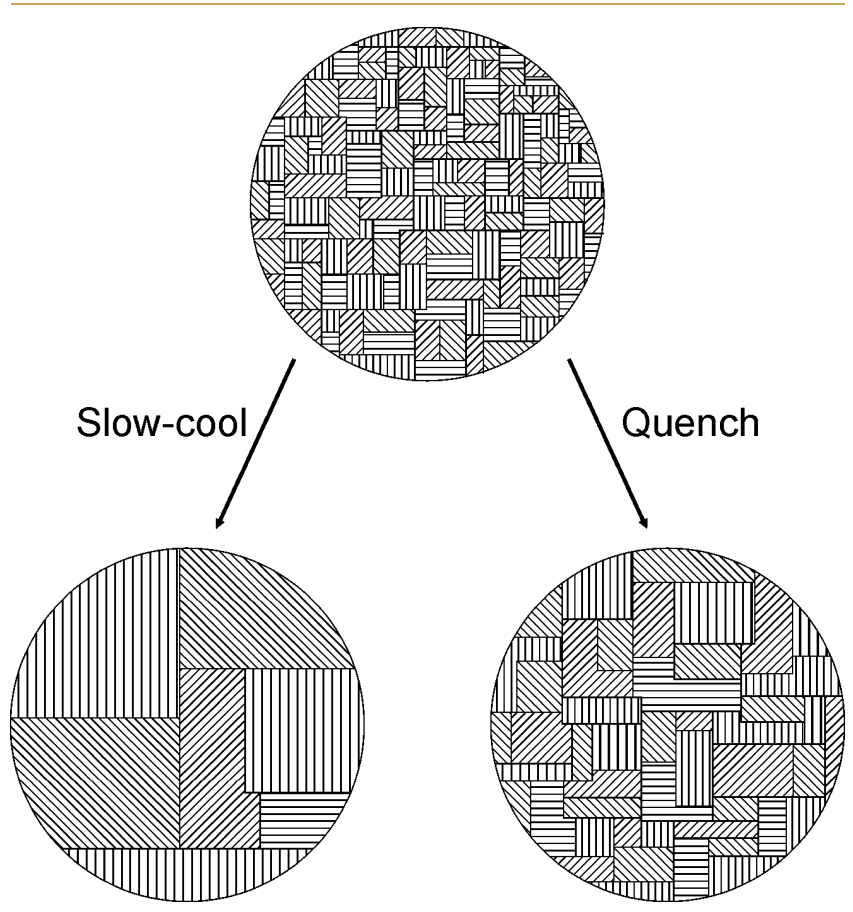

Figure 5. Schematic illustrating domain growth in $\mathrm{LaSrNiRuO}_{6}$. On cooling below the structural phase transition, a patch work of small domains forms (top). These domains grow at different rates dependent on the cooling rate of the sample.

This patchwork of domains is energetically unfavorable. First, this is because the interfaces between domains are energetically expensive, as there are discontinuities in the cooperative tilts of the $\mathrm{NiO}_{6}$ and $\mathrm{RuO}_{6}$ units. A second unfavorable feature comes from the mismatch in the unit cell distortions of the low- and high-temperature phases. This arises because, relative to the aristotype, $a^{0} a^{0} a^{0}$ undistorted Fm-3m symmetry phase of $\mathrm{LaSrNiRuO}_{6}$, the $R-3$ high- temperature phase has a unit cell that is expanded along one of the [111] directions. However, the low-temperature $P 2_{1} / n$ phase has a unit cell that is expanded along [110] and contracted along $[1-10]$. Thus, conversion from the $R-3$ phase to the $P 2_{1} / n$ phase leads to a change in the shape or aspect ratio of the underlying perovskite framework. However, the presence of large numbers of small domains hampers this aspect ratio change, and as a result, the crystal domains formed are under considerable strain.

To lower the free energy of the system, some of the crystal domains will grow, at the expense of others over time, to relieve the lattice strain and minimize the area of the interfaces between domains. However, domain growth has a significant activation energy, and as a result, the rate of domain growth is temperature-dependent, dropping to zero below some "freezing" temperature. Quenched samples cool quickly, so they spend little time in the "domain growth temperature window", which has the phase transition as its upper limit and the domain freezing temperature as its lower limit. As a result, samples quenched from temperatures just above the phase transition temperature only undergo modest domain growth as shown in Figure 5. In contrast, slow-cooled samples spend far longer at temperatures where domain growth can occur and as a result undergo extensive domain growth. Thus, if a sintered pellet of $\mathrm{LaSrNiRuO}_{6}$ is either quenched of slow-cooled, a polycrystalline pellet with either small, highly strained domains or larger, less strained domains will be formed. When these pellets are then ground to powder, they will tend to fracture along the boundaries between domains, so the quenched pellet will be ground into a powder with smaller average particle/ domain size and higher lattice strain than the slow-cooled pellet, accounting for the observed difference in microstructure between slow-cooled and quenched samples.

\section{Structural Phase Transition of LaSrNiRuO 6}

To further investigate the influence of sample quenching, SXRD data were collected as a function of temperature from a slow-cooled sample of $\mathrm{LaSrNiRuO}_{6}$ and a sample quenched from $375{ }^{\circ} \mathrm{C}$, which were heated at $12{ }^{\circ} \mathrm{C} \mathrm{min}{ }^{-1}$ from 100 to $950{ }^{\circ} \mathrm{C}$ and then cooled at the same rate back to $100^{\circ} \mathrm{C}$. Each data set collected was fitted by structural models describing the $P 2_{1} / n$ and $R-3$ phases of $\mathrm{LaSrNiRuO}_{6}$ to determine the fraction of each phase in the sample at a given temperature.

The data in Figure 6 show that on heating, the slow-cooled and quenched samples begin transforming from $P 2_{1} / n$ to $R-3$ symmetry at 350 and $315^{\circ} \mathrm{C}$, respectively. However, this phase transition spans a wide temperature range, with complete conversion to the $R-3$ phase not achieved until 760 and $730{ }^{\circ} \mathrm{C}$ for the slow-cooled and quenched samples, respectively, with a two-phase mixture existing between these temperatures. Data collected on cooling show the phase transition exhibits significant thermal hysteresis, with the reverse $R-3$ to $P 2_{1} / n$ transition not beginning until 605 and $680{ }^{\circ} \mathrm{C}$ for the slowcooled and quenched samples, respectively, and complete conversion back to the $P 2_{1} / n$ phase not achieved until 325 and $315{ }^{\circ} \mathrm{C}$, respectively.

The broad two-phase region observed and the large thermal hysteresis exhibited by the $P 2_{1} / n$-to- $R-3$ phase transition is consistent with the presence of a large energetic barrier ("activation energy"), which needs to be overcome during the structural change. The smaller thermal hysteresis observed for the quenched sample, compared to the slow-cooled sample, suggests that samples with smaller domain sizes and larger 


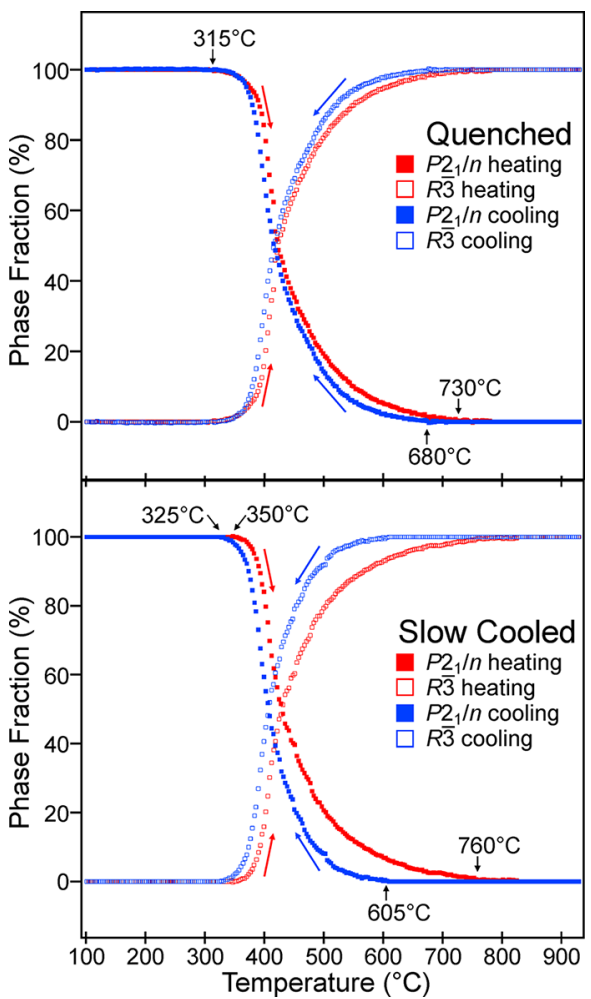

Figure 6. Plot of phase fraction against temperature for the structural phase transition of slow-cooled and quenched samples of LaSrNiR$\mathrm{uO}_{6}$ determined by variable temperature SXRD data. Indicated temperatures mark the beginning and end of the phase transition on heating and cooling.

lattice strain have a smaller $P 2_{1} / n$-to- $R$ - 3 phase-transition energy barrier. In addition, the lower onset temperature for the phase transition of the quenched sample suggests that the enhanced crystal strain and/or smaller domain size could destabilize the $P 2_{1} / n$ phase with respect to the $R-3$ phase, leading to lower phase transition onset temperature.

It should be noted that a comparison of the SXRD data collected at $100{ }^{\circ} \mathrm{C}$ before and after the heating/cooling cycle shown in Figure 6 reveals that these data are identical, indicating the microstructural differences between the slowcooled and quenched samples are preserved through this heating cycle. In contrast, SXRD data collected after annealing the quenched sample at $360{ }^{\circ} \mathrm{C}$ (the temperature of the reduction reaction) for 2 days revealed a slight sharpening of the diffraction peaks due to a decrease in lattice strain but no observable change in domain size.

The extensive two-phase region and thermal hysteresis exhibited by $\mathrm{LaSrNiRuO}_{6}$ at the $P 2_{1} / n$ to $R-3$ phase transition is consistent with a large activation energy associated with the changes in the cooperative tilting of the $\mathrm{MO}_{6}$ units necessary to change from an $a^{-} a^{-} a^{-}$to an $a^{-} a^{-} c^{+}$tilting pattern or to move a domain wall between differently oriented $a^{-} a^{-} c^{+}$ domains and further suggests that the structural change at the phase transition is kinetically slow. It is likely that this large activation energy enhances the effect of sample quenching on the microstructure of $\mathrm{LaSrNiRuO}_{6}$.

Influence of the Microstructure on the Rate and Extent of Reduction

The differing phase-transition behavior observed for quenched and slow-cooled samples of $\mathrm{LaSrNiRuO}_{6}$ suggests a mecha- nism by which the microstructure of samples can influence the rate and extent of the reduction reaction that forms $\mathrm{LaSrNiRuO}_{4}$. Diffraction data collected during the reduction of $\mathrm{LaSrNiRuO}_{6}$ (Figure 2) reveal that the only Ni/Ru phases observable during the reaction are pseudocubic $\mathrm{LaSrNiRuO}_{6-x}$ and pseudotetragonal $\mathrm{LaSrNiRuO}_{4+x}$, with no evidence for intermediate " $\mathrm{LaSrNiRuO}_{5}$ " type phases. This indicates that $\mathrm{LaSrNiRuO}_{6}$ and $\mathrm{LaSrNiRuO}_{4}$ are close to being "line phases" and that $\mathrm{LaSrNiRuO}_{6}$ is converted directly into $\mathrm{LaSrNiRuO}_{4}$ without forming an appreciable concentration of material with an intermediate oxygen stoichiometry. Thus, we would expect that as oxygen is deintercalated from a particle/domain of $\mathrm{LaSrNiRuO}_{6}$, a layer of $\mathrm{LaSrNiRuO}_{4}$ will form at the particle/ domain surface, demarcated by a relatively sharp interface from the unreduced $\mathrm{LaSrNiRuO}_{6}$ in the rest of the particle/domain, as shown in Figure $7 \mathrm{a}$.

Thin film studies of the reduction of $\mathrm{SrFeO}_{3-\delta}$ to $\mathrm{SrFeO}_{2}{ }^{19,20}$ and $\mathrm{LaNiO}_{3}$ to $\mathrm{LaNiO}_{2}{ }^{21}$ reveal that the infinitelayer phases synthesized by reduction of perovskite hosts tend to form with their $c$-axis aligned perpendicular to the particle/ domain surface, rather than parallel to it. Thus, we would expect that as oxygen is deintercalated from a particle/domain of $\mathrm{LaSrNiRuO}_{6}$, a layer of $\mathrm{LaSrNiRuO}_{4}$ will form at the particle/domain surface aligned with its $c$-axis perpendicular to the particle/domain surface, as shown in Figure 5.

The reduction of $\mathrm{LaSrNiRuO}_{6}(a=5.575 \AA, b=5.542 \AA, c=$ $\left.7.848 \AA, \beta=90.0^{\circ}\right)^{16}$ to $\mathrm{LaSrNiRuO}_{4}(a=5.652 \AA, b=5.652$ $\left.\AA, c=6.915 \AA, \beta=90.5^{\circ}\right)^{13}$ leads to a dramatic change to the lattice parameters of the material: a contraction of the $c$-axis of $\sim 11.9 \%$ and an expansion in the area of the $a b$-plane of $\sim 3.3 \%$ resulting in a decrease in cell volume of $\sim 9.1 \%$. Therefore, even with a $c$-axis aligned region of $\mathrm{LaSrNiRuO}_{4}$ (Figure 7a), there will be a very large, energetically unfavorable lattice strain present at the interface between the regions of $\mathrm{LaSrNiRuO}_{4}$ and $\mathrm{LaSrNiRuO}_{6}$ due to the large mismatch in lattice parameters of the two phases.

We propose that this large interfacial lattice strain is the origin of the microstructure-dependent reactivity of LaSrNiR$\mathrm{uO}_{6}$, because as a particle/domain of $\mathrm{LaSrNiRuO}_{6}$ is reduced to $\mathrm{LaSrNiRuO}_{4}$, the large lattice strain that is established at the reaction interface opposes the reduction of $\mathrm{LaSrNiRuO}_{6}$ and thus appears to add to the activation energy of the reduction reaction. Small particles/domains of $\mathrm{LaSrNiRuO}_{6} / \mathrm{LaSrNiR}$ $\mathrm{uO}_{4}$ are more flexible than larger particles/domains, and this enhanced plasticity results in a smaller interfacial strain contribution to the activation energy of the reduction reaction. As a result, small domains/particles will have a lower activation energy for reduction than larger domains/particles, as illustrated in Figure $7 \mathrm{~b}$.

As noted above, particles/domains of $\mathrm{LaSrNiRuO}_{6}$ that have high lattice strains, due to rapid quenching, are less stable than less strained domains. This destabilization of $\mathrm{LaSrNiRuO}_{6}$ via lattice strain will also act to reduce the apparent activation energy of the $\mathrm{LaSrNiRuO}_{6}$-to- $\mathrm{LaSrNiRuO}_{4}$ reduction reaction (just as it does for the $P 2_{1} / n$-to- $R-3$ phase transition) as illustrated in Figure $7 \mathrm{~b}$.

Given that the activation energy of the $\mathrm{LaSrNiRuO}_{6}$-to$\mathrm{LaSrNiRuO}_{4}$ reaction is strongly affected by particle/domain size and lattice strain, we can separate the reduction-activation energy into two parts: an "intrinsic" activation energy arising from the chemical transformation (i.e., the reduction-activation energy of an unstrained macroscopic single crystal) and a "microstructural" contribution that lowers the activation 
a)

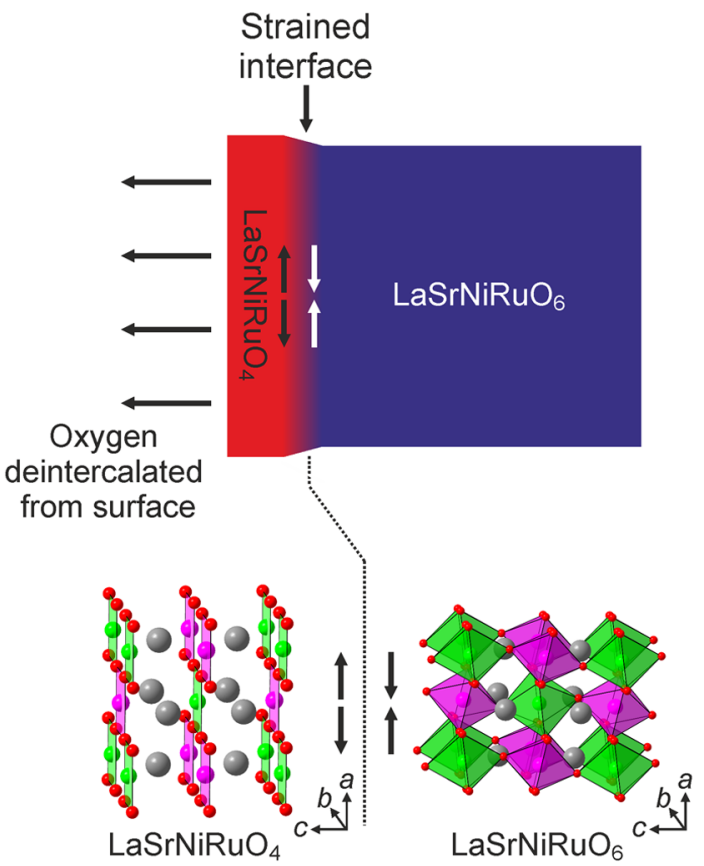

b)

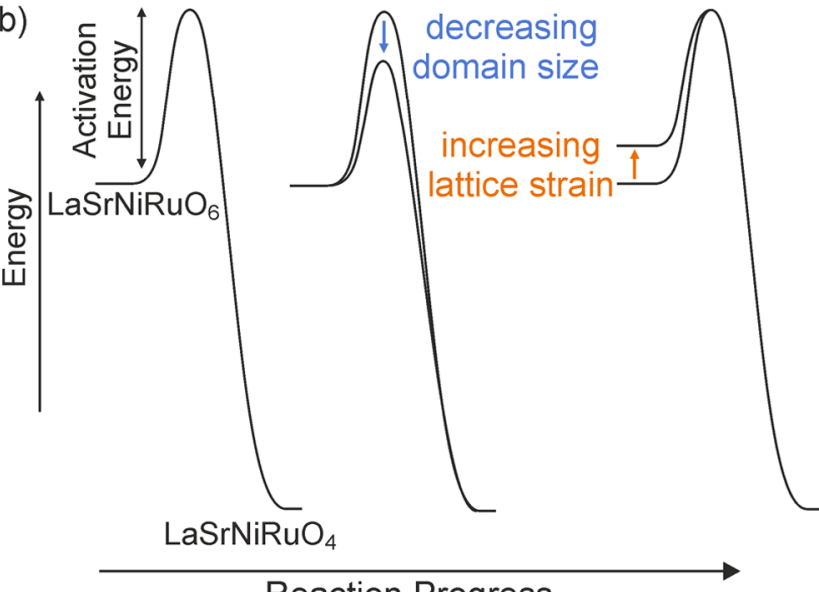

c)

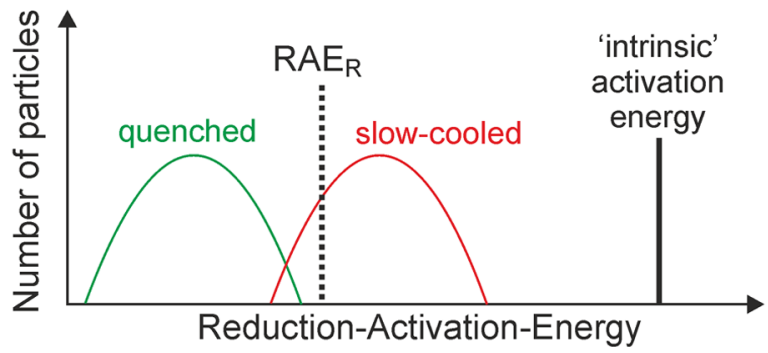

Figure 7. (a) Schematic diagram of the interface between $\mathrm{LaSrNiRuO}_{6-x}$ (blue) and $\mathrm{LaSrNiRuO}_{4+x}$ (red) formed during the reduction of a crystalline domain. (b) Schematic diagram showing how particle/domain size and lattice strain can lower the reductionactivation energy. (c) Schematic diagram showing how the influence of microstructural effects on the reduction-activation energy can lead to the observed differences in the reactivity of quenched and slowcooled samples of $\mathrm{LaSrNiRuO}_{6}$.

energy by the processes described above. Any powder sample of $\mathrm{LaSrNiRuO}_{6}$ contains a population of powder grains with a range of particle/domain sizes and lattice strains that will lower the reduction-activation energy by different amounts. The reduction-activation energy of a powder sample will therefore span a range of values dependent on the distribution of particle/domain sizes and lattice strains of particles within sample. Figure $7 \mathrm{c}$ shows a schematic of the range of reductionactivation energies for a slow-cooled and a quenched sample of $\mathrm{LaSrNiRuO}_{6}$, with the quenched sample exhibiting a smaller average reduction-activation energy due to its smaller average particle/domain size and higher average lattice strain.

At a given reduction temperature, $T_{R}$, there is a set amount of thermal energy available, and thus, there is maximum value of activation energy, $\mathrm{RAE}_{\mathrm{R}}$, which can be overcome at this temperature. The differing reactivity of slow-cooled and quenched samples of $\mathrm{LaSrNiRuO}_{6}$ is consistent with the situation shown in Figure $7 \mathrm{c}$. The large lattice strain and small particle/domain size of the quenched sample lower the reduction-activation energy of all particles in the sample below the $\mathrm{RAE}_{\mathrm{R}}$ at $360{ }^{\circ} \mathrm{C}$, allowing complete and rapid conversion to $\mathrm{LaSrNiRuO}_{4}$. In contrast, the smaller lattice strain and larger particle/domain size of the slow-cooled sample lead to a smaller lowering of the reduction-activation energy, so only a fraction of the particles in the sample have reduction-activation-energies below the $\mathrm{RAE}_{\mathrm{R}}$ at $360{ }^{\circ} \mathrm{C}$, so only partial reduction of the sample occurs.

In many topochemical reactions, a lack of reactivity can be overcome by simply raising the reaction temperature. However, in the case of the $\mathrm{LaSrNiRuO}_{6}$-to- $\mathrm{LaSrNiRuO}_{4}$ reaction, raising the reaction temperature leads to the onset of nontopochemical decomposition reactions. Thus, we can see that in the absence of "microstructural activation" it is not possible to prepare single-phase samples of $\mathrm{LaSrNiRuO}_{4}$ via reduction of $\mathrm{LaSrNiRuO}_{6}$.

This situation highlights a general requirement of topochemical transformations: that there is a significant difference in the reaction rate (and therefore activation energy) between the desired topochemical process (e.g., anion deintercalation) and competing nontopochemical processes (e.g., structural rearrangement/decomposition). There are many cases, such as the $\mathrm{LaSrNiRuO}_{6}$-to- $\mathrm{LaSrNiRuO}_{4}$ transformation, where the difference in rate/activation energy is insufficient to allow the preparation of single-phased samples of the topochemically manipulated product phase. Fortunately, in the $\mathrm{LaSrNiRuO}_{6}$ to- $\mathrm{LaSrNiRuO}{ }_{4}$ case, there is a convenient structural phase transition that allows the microstructure of the material to be manipulated to "activate" the topochemical reaction, but there are many instances where this type of activation is not possible. There are however other methods of achieving analogous activations of topochemical processes. For example, the "hardsoft" reaction sequence developed by Attfield ${ }^{22,23}$-in which a "host" phase is prepared in a high-temperature, high-pressure reaction, quenched to ambient conditions, and then further transformed by a topochemical reaction-would be expected to benefit from enhanced reactivity due to the increased lattice strain present in the metastable host phase at ambient pressure. More generally, strain can be applied to "host" structures by depositing them as thin films on to substrates with slight lattice mismatches. Indeed, it is generally observed that thin films react faster and at lower temperatures than bulk powder samples, ${ }^{20,21,24-27}$ providing further support for the microstructural activation of solid-state topochemical reactions.

This microstructural activation of anion-focused topochemical reactions mirrors the extensive observations of microstructural effects in cation intercalation/deintercalation reactions, where it is observed that particle size ${ }^{28-32}$ and the buildup of lattice $\operatorname{strain}^{33,34}$ during intercalation/deintercala- 
tion processes can affect not only the rates and extents of chemical conversion but also the pathways adopted.

\section{CONCLUSIONS}

The enhanced reactivity of quenched samples of $\mathrm{LaSrNiRuO}_{6}$, compared to analogous slow-cooled samples, can be attributed to the presence of a structural phase transition at $T \approx 400{ }^{\circ} \mathrm{C}$, which manipulates the microstructure of the double perovskite oxide so that quenched powder samples of $\mathrm{LaSrNiRuO}_{6}$ have smaller average particle/domain sizes and higher lattice strains than slow-cooled samples. These microstructural features act to lower the activation energy of the topochemical reduction reaction that converts $\mathrm{LaSrNiRuO}_{6}$ into $\mathrm{LaSrNiRuO}_{4}$, allowing the rapid reduction of quenched samples of $\mathrm{LaSrNiRuO}_{6}$ at $360{ }^{\circ} \mathrm{C}$ but not slow-cooled samples. The sensitivity of the progress of the reduction reaction to the thermal history of the $\mathrm{LaSrNiRuO}_{6}$ sample indicates that topochemical reduction reactions can be microstructurally activated, potentially expanding the range of solid phases that can be prepared by this type of process.

\section{ASSOCIATED CONTENT}

\section{Supporting Information}

The Supporting Information is available free of charge at https://pubs.acs.org/doi/10.1021/acsorginorgau.1c00030.

Complete description of the chemical, structural, and microstructural characterization of quenched and slowcooled $\mathrm{LaSrNiRuO}_{6}$. Details of SEM particle-size analysis (PDF)

\section{AUTHOR INFORMATION}

\section{Corresponding Author}

Michael A. Hayward - Department of Chemistry, University of Oxford, Inorganic Chemistry Laboratory, Oxford OX1 3QR, U.K.; (1) orcid.org/0000-0002-6248-2063;

Email: michael.hayward@chem.ox.ac.uk

\section{Authors}

Zhilin Liang - Department of Chemistry, University of Oxford, Inorganic Chemistry Laboratory, Oxford OX1 3QR, U.K.

Midori Amano Patino - Department of Chemistry, University of Oxford, Inorganic Chemistry Laboratory, Oxford OX1 $3 Q R$, U.K.

Mylène Hendrickx - EMAT, University of Antwerp, B-2020 Antwerp, Belgium

Joke Hadermann - EMAT, University of Antwerp, B-2020

Antwerp, Belgium; (1) orcid.org/0000-0002-1756-2566

Complete contact information is available at:

https://pubs.acs.org/10.1021/acsorginorgau.1c00030

\section{Author Contributions}

The manuscript was written through contributions of all authors.

Notes

The authors declare no competing financial interest.

\section{ACKNOWLEDGMENTS}

Experiments at the Diamond Light Source were performed as part of the Block Allocation Group award "Oxford Solid State
Chemistry BAG to probe composition-structure-property relationships in solids" (CY25166). Z.L. and M.A.H. thank the EPSRC (EP/T027991/1) for funding.

\section{REFERENCES}

(1) Hayward, M. A. Soft chemistry synthesis of oxides. Comprehensive Inorganic Chemistry II 2013, 2, 417-453.

(2) Hayward, M. A.; Green, M. A.; Rosseinsky, M. J.; Sloan, J. Sodium hydride as a powerful reducing agent for topotactic oxide deintercalation: synthesis and characterisation of the nickel (I) oxide $\mathrm{LaNiO}_{2}$. J. Am. Chem. Soc. 1999, 121, 8843-8854.

(3) Tsujimoto, Y.; Tassel, C.; Hayashi, N.; Watanabe, T.; Kageyama, H.; Yoshimura, K.; Takano, M.; Ceretti, M.; Ritter, C.; Paulus, W. Infinite-layer iron oxide with a square-plannar coordination. Nature 2007, 450, 1062-1065.

(4) Seddon, J.; Suard, E.; Hayward, M. A. Topotactic reduction of $\mathrm{YBaCo}_{2} \mathrm{O}_{5}$ and $\mathrm{LaBaCo}_{2} \mathrm{O}_{5}$ : square-planer $\mathrm{Co}(\mathrm{I})$ in an extended oxide. J. Am. Chem. Soc. 2010, 132, 2802-2810.

(5) Denis Romero, F.; Leach, A.; Möller, J. S.; Foronda, F.; Blundell, S.; Hayward, M. A. Strontium vanadium oxide-hydrides: 'squareplanar' two-electron phases. Angew. Chem., Int. Ed. 2014, 53, 75567559 .

(6) Zhang, R.; Read, G.; Lang, F.; Lancaster, T.; Blundell, S. J.; Hayward, M. A. $\mathrm{La}_{2} \mathrm{SrCr}_{2} \mathrm{O}_{7} \mathrm{~F}_{2}$ - A Ruddlesden-Popper Oxyfluoride Containing Octahedrally Coordinated $\mathrm{Cr}^{4+}$ Centers. Inorg. Chem. 2016, 55, 3169-3174.

(7) Luo, K.; Johnson, R. D.; Tran, T. T.; Halasyamani, P. S.; Radaelli, P. G.; Hayward, M. A. $\mathrm{Ba}_{2} \mathrm{YFeO}_{5.5}$ : A Ferromagnetic Pyroelectric Phase Prepared by Topochemical Oxidation. Chem. Mater. 2013, 25 (9), 1800-1808.

(8) Denis Romero, F.; Burr, S. J.; McGrady, J. E.; Gianolio, D.; Cibin, G.; Hayward, M. A. $\mathrm{SrFe}_{0.5} \mathrm{Ru}_{0.5} \mathrm{O}_{2}$ : sqaure-planar $\mathrm{Ru}^{2+}$ in an extended oxide. J. Am. Chem. Soc. 2013, 135, 1838-1844.

(9) Page, J. E.; Morgan, H. W. T.; Zeng, D.; Manuel, P.; McGrady, J. E.; Hayward, M. A. $\mathrm{Sr}_{2} \mathrm{FeIrO}_{4}$ : square-planar $\mathrm{Ir}(\mathrm{II})$ in an extended oxide. Inorg. Chem. 2018, 57, 13577-13585.

(10) Jin, L.; Lane, M.; Zeng, D.; Kirschner, F. K. K.; Lang, F.; Manuel, P.; Blundell, S. J.; McGrady, J. E.; Hayward, M. A. $\mathrm{LaSr}_{3} \mathrm{NiRuO}_{4} \mathrm{H}_{4}$ : a $4 \mathrm{~d}$ transition-metal oxide-hydride containing metal hydride sheets. Angew. Chem. Angew. Chem., Int. Ed. 2018, 57, 5025-5028.

(11) Xu, Z.; Palgrave, R. G.; Hayward, M. A. $\mathrm{LaSrCo}_{0.5} \mathrm{Rh}_{0.5} \mathrm{O}_{3.25}$ and $\mathrm{LaSrNi}_{0.5} \mathrm{Rh}_{0.5} \mathrm{O}_{3.25}$ : Topochemically Reduced, Mixed Valence $\mathrm{Rh}(\mathrm{I}) /$ $\mathrm{Rh}(\mathrm{III})$ Oxides. Inorg. Chem. 2020, 59, 13767-13773.

(12) Hayward, M. A. Synthesis and Magnetism of Extended Solids Containing Transition-Metal Cations in Square-Planar, MO4 Coordination Sites. Inorg. Chem. 2019, 58 (18), 11961-11970.

(13) Amano Patino, M.; Zeng, D.; Bower, R.; McGrady, J. E.; Hayward, M. A. Coupled electronic and magnetic phase transition in the infinite-layer phase $\mathrm{LaSrNiRuO}_{4}$. Inorg. Chem. 2016, 55 (17), 9012-9016.

(14) O'Malley, M.; Lockett, M. A.; Hayward, M. A. Anion vacancy ordering in $\mathrm{Sr}_{7} \mathrm{Mn}_{4} \mathrm{O}_{15-\mathrm{x}}$ phases. J. Solid State Chem. 2007, 180, 28512858.

(15) Coelho, A. A. TOPAS Academic: General profile and Structure Analysis Software For Powder Diffraction Data; 2016.

(16) Gateshki, M.; Igartua, J. M. Crystal structures and phase transitions of the double perovskite oxides $\mathrm{SrLaCuRuO}_{6}$ and SrLaNiRuO 6 . Mater. Res. Bull. 2003, 38 (14), 1893-1900.

(17) Glazer, A. M. Classification of tilted Octahedra in Perovskites. Acta Crystallogr., Sect. B: Struct. Crystallogr. Cryst. Chem. 1972, 28 (11), 3384-3392.

(18) Woodward, P. M. Octahedral tilting in perovskites. 1. Geometrical considerations. Acta Crystallogr., Sect. B: Struct. Sci. 1997, 53, 32-43.

(19) Inoue, S.; Kawai, M.; Ichikawa, N.; Kageyama, H.; Paulus, W.; Shimakawa, Y. Anisotropic oxygen diffusion at low temperature in perovskite-structure iron oxides. Nat. Chem. 2010, 2 (3), 213-217. 
(20) Inoue, S.; Kawai, M.; Shimakawa, Y.; Mizumaki, M.; Kawamura, N.; Watanabe, T.; Tsujimoto, Y.; Kageyama, H.; Yoshimura, K. Single-crystal epitaxial thin films of $\mathrm{SrFeO}_{2}$ with $\mathrm{FeO}_{2}$ "infinite layers". Appl. Phys. Lett. 2008, 92 (16), 161911.

(21) Kawai, M.; Matsumoto, K.; Ichikawa, N.; Mizumaki, M.; Sakata, O.; Kawamura, N.; Kimura, S.; Shimakawa, Y. Orientation Change of an Infinite-Layer Structure $\mathrm{LaNiO}_{2}$ Epitaxial Thin Film by Annealing with $\mathrm{CaH}_{2}$. Cryst. Growth Des. 2010, 10 (5), 2044-2046.

(22) Arevalo-Lopez, A. M.; Liang, B.; Senn, M. S.; Murray, C.; Tang, C.; Attfield, J. P. Hard-soft synthesis of a new series of vacancyordered perovskites, $\mathrm{CaCrO}_{3-\mathrm{x}}$. J. Mater. Chem. C 2014, 2 (44), 93649367.

(23) Arevalo-Lopez, A. M.; Srinath, A.; Attfield, J. P. Hard-soft chemistry of $\mathrm{Sr}_{1-\mathrm{x}} \mathrm{Ca}_{\mathrm{x}} \mathrm{CrO}_{3-\mathrm{d}}$ solid solutions. Materials Chemistry Frontiers 2017, 1 (1), 172-175.

(24) Li, D. F.; Lee, K.; Wang, B. Y.; Osada, M.; Crossley, S.; Lee, H. R.; Cui, Y.; Hikita, Y.; Hwang, H. Y. Superconductivity in an infinitelayer nickelate. Nature 2019, 572 (7771), 624-627.

(25) Katayama, T.; Chikamatsu, A.; Yamada, K.; Shigematsu, K.; Onozuka, T.; Minohara, M.; Kumigashira, H.; Ikenaga, E.; Hasegawa, T. Epitaxial growth and electronic structure of oxyhydride $\mathrm{SrVO} 2 \mathrm{H}$ thin films. J. Appl. Phys. 2016, 120 (8), 085305.

(26) Namba, M.; Takatsu, H.; Yoshimune, W.; Daniel, A.; Itoh, S.; Terashima, T.; Kageyama, H. A Partial Anion Disorder in SrVO2H Induced by Biaxial Tensile Strain. Inorganics 2020, 8, 26.

(27) Sakaguchi, T.; Kobayashi, Y.; Yajima, T.; Ohkura, M.; Tassel, C.; Takeiri, F.; Mitsuoka, S.; Ohkubo, H.; Yamamoto, T.; Kim, J. E.; Tsuji, N.; Fujihara, A.; Matsushita, Y.; Hester, J.; Avdeev, M.; Ohoyama, K.; Kageyama, H. Oxyhydrides of $(\mathrm{Ca}, \mathrm{Sr}, \mathrm{Ba}) \mathrm{TiO}_{3}$ perovskite solid solutions. Inorg. Chem. 2012, 51 (21), 11371-11376.

(28) Liu, H.; Grey, C. P. Influence of particle size, cycling rate and temperature on the lithiation process of anatase TiO2. J. Mater. Chem. A 2016, 4 (17), 6433-6446.

(29) Maier, J. Nanoionics: ionic charge carriers in small systems. Phys. Chem. Chem. Phys. 2009, 11 (17), 3011-3022.

(30) Wagemaker, M.; Borghols, W. J. H.; Mulder, F. M. Large impact of particle size on insertion reactions. A case for anatase LixTiO2. J. Am. Chem. Soc. 2007, 129 (14), 4323-4327.

(31) Burch, D.; Bazant, M. Z. Size-Dependent Spinodal and Miscibility Gaps for Intercalation in Nanoparticles. Nano Lett. 2009, 9 (11), 3795-3800.

(32) Meethong, N.; Huang, H. Y. S.; Carter, W. C.; Chiang, Y. M. Size-dependent lithium miscibility gap in nanoscale Li1-xFePO4. Electrochem. Solid-State Lett. 2007, 10 (5), A134-A138.

(33) Meethong, N.; Huang, H. Y. S.; Speakman, S. A.; Carter, W. C.; Chiang, Y. M. Strain accommodation during phase transformations in olivine-based cathodes as a materials selection criterion for highpower rechargeable batteries. Adv. Funct. Mater. 2007, 17 (7), 11151123

(34) Van der Ven, A.; Garikipati, K.; Kim, S.; Wagemaker, M. The Role of Coherency Strains on Phase Stability in LixFePO4: Needle Crystallites Minimize Coherency Strain and Overpotential. J. Electrochem. Soc. 2009, 156 (11), A949-A957.

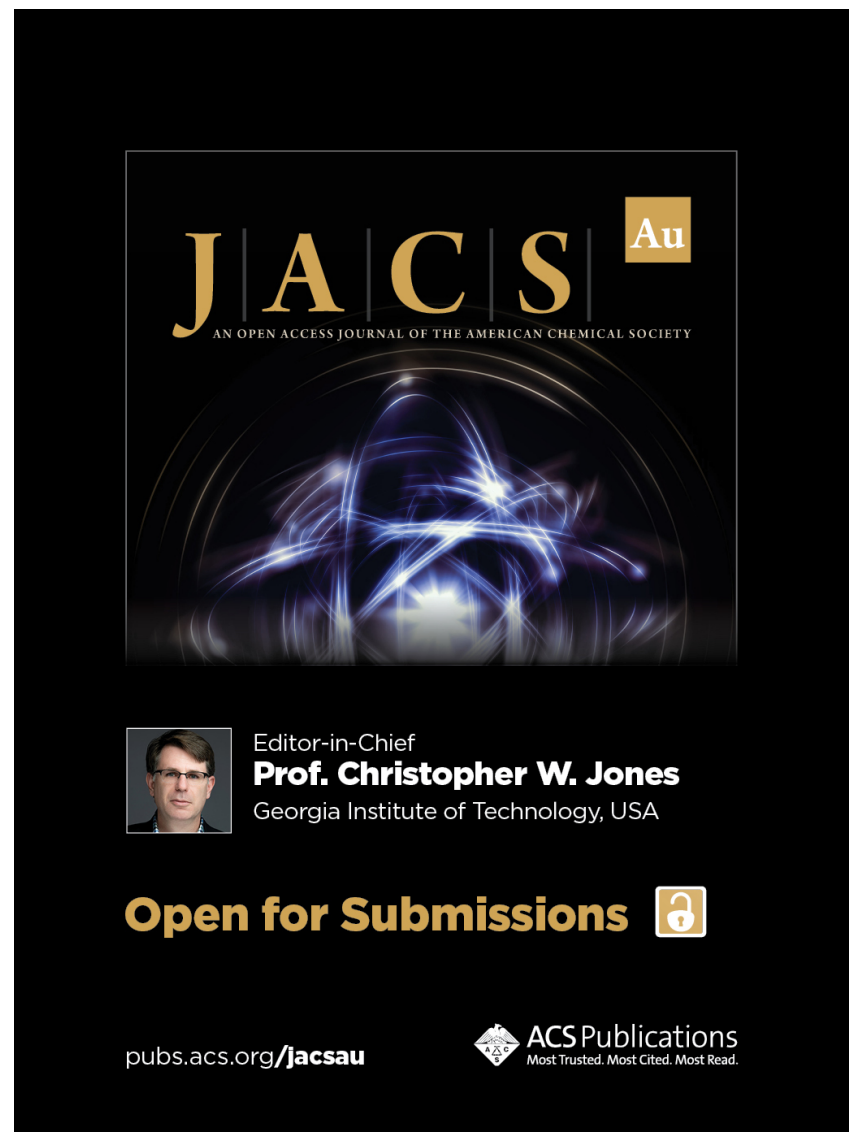

